\title{
SIGN CHANGES IN HARMONIC ANALYSIS ON REDUCTIVE GROUPS
}

BY

ROBERT E. KOTTWITZ ${ }^{1}$

\begin{abstract}
Let $G$ be a connected reductive group over a field $F$. In this note the author constructs an element $e(G)$ of the Brauer group of $F$. The square of this element is trivial. For a local field, $e(G)$ may be regarded as an element of $\{ \pm 1\}$ and is needed for harmonic analysis on reductive groups over that field. For a global field there is a product formula.
\end{abstract}

The purpose of this note is to provide a cohomological interpretation of the sign changes in harmonic analysis on reductive groups over local fields that are caused by inner twistings. The best understood example occurs in the work of Shelstad [8] on inner twistings of real groups. Theorem 6.3 of that paper gives the following character identity:

$$
\chi_{\varphi^{\prime}}\left(\gamma^{\prime}\right)=(-1)^{q\left(G^{\prime}\right)-q(G)} \chi_{\varphi}(\gamma) .
$$

Here $G$ is a connected reductive group over $\mathbf{R}, G^{\prime}$ is its quasi-split inner form, $\chi_{\varphi^{\prime}}$ and $\chi_{\varphi}$ are stabilized characters of matched tempered $L$-packets of $G^{\prime}(\mathbf{R})$ and $G(\mathbf{R})$, and $\gamma^{\prime}$ and $\gamma$ are matched regular semisimple elements of $G^{\prime}(\mathbf{R})$ and $G(\mathbf{R})$. The sign change in the character identity is $(-1)^{q\left(G^{\prime}\right)-q(G)}$ where $q(G)$ is one half of the dimension of the symmetric space attached to $G$ (more precisely, attached to the simply connected cover of the derived group of $G)$. Although $q(G)$ may be only half integral, the difference $q\left(G^{\prime}\right)-q(G)$ is always integral.

It is reasonable to expect an analogous character identity for groups $G$ over a nonarchimedean local field $F$. We can determine what the sign change must be by considering the Steinberg characters of $G(F)$ and $G^{\prime}(F)$, since these should be related by the character identity. Let $r(G)$ denote the $F$-rank of the derived group of $G$; the Steinberg character of $G(F)$ has value $(-1)^{r(G)}$ on the elliptic regular set of $G(F)$, and hence the sign change in the character identity must be $(-1)^{r\left(G^{\prime}\right)-r(G)}$. There are two groups for which the character identity has been proved: the multiplicative group of a central division algebra of dimension $d^{2}$ over $F$ for $d=2$ (see Proposition 15.5 on p. 484 of [4]) and $d=3$ (see Theorem 1 of [1]).

Later in this paper we will use a cohomological construction to define a sign $e(G)= \pm 1$ for any connected reductive group $G$ over a local field. Let $G^{\prime}$ denote a quasi-split inner form of $G$. We will show that $e(G)=(-1)^{r\left(G^{\prime}\right)-r(G)}$ when the base

Received by the editors May 10, 1982.

1980 Mathematics Subject Classification. Primary 22E50.

' Partially supported by the National Science Foundation under Grant MCS 78-02331. 
field is nonarchimedean, $e(G)=(-1)^{q\left(G^{\prime}\right)-q(G)}$ when the base field is $\mathbf{R}$, and $e(G)=1$ when the base field is $\mathbf{C}$. For a group $G$ over a global field we will prove a product formula $\Pi_{v} e\left(G_{v}\right)=1$; the product is taken over all places $v$ of the global field, all but a finite number of terms in the product being 1 . This product formula is consistent with our expectation that no sign changes occur when comparing the characters (as distributions) of automorphic representations of $G, G^{\prime}$ (since no sign changes occur in going from the elliptic regular terms of the trace formula for $G$ to those for $G^{\prime}$ ).

Changes of sign also occur in the matching of functions by orbital integrals. For example let $E / F$ be a cyclic extension of local fields, let $G=G L_{2}$, and consider the matching of functions on $G(E)$ and $G(F)$ that arises in the theory of base change. For our purposes the best reference is [5]. There is a sign $\varepsilon(\gamma)$ in the formula $\left(A^{\prime}\right)$ of that article:

$$
\int_{G_{\gamma}(F) \backslash G(F)} f\left(g^{-1} \gamma g\right) d g / d t=\varepsilon(\gamma) \int_{G_{\delta}^{o}(E) \backslash G(E)} \varphi\left(g^{-\sigma} \delta g\right) d g_{E} / d t^{\prime} .
$$

For semisimple $\gamma$ the sign can be explained in the following way. The element $\delta \in G(E)$ can be used to define an inner form $G_{\gamma}^{\prime}$ of $G_{\gamma}$ such that $G_{\gamma}^{\prime}(F)=G_{\delta}^{\sigma}(E)$. Since we are considering semisimple $\gamma, G_{\gamma}$ is reductive. Since $G$ is $G L_{2}, G_{\gamma}$ is connected as well. It turns out that $\varepsilon(\gamma)$ is equal to $e\left(G_{\gamma}\right) / e\left(G_{\gamma}^{\prime}\right)$.

We can give another example with a similar result. Let $F$ be a local field, $D$ a quaternion algebra over $F, G$ the group over $F$ associated to $D^{\times}, G^{\prime}$ the quasi-split inner form of $G$, namely $G L_{2}$. Let $\bar{F}$ denote an algebraic closure of $F$ and choose an isomorphism $D \otimes_{F} \bar{F} \rightarrow M_{2}(\bar{F})$. This induces an isomorphism $\varphi: G \rightarrow G^{\prime}$ over $\bar{F}$. For every $x \in G(F)$ there exists $y \in G^{\prime}(F)$ such that $\varphi(x)$ and $y$ are conjugate in $G^{\prime}(\bar{F})$, and the conjugacy class of $y$ in $G^{\prime}(F)$ is determined uniquely by the conjugacy class of $x$ in $G(F)$. It can be shown that for every smooth compactly supported function $f$ on $G(F)$ there exists a smooth compactly supported function $f^{\prime}$ on $G^{\prime}(F)$ such that

$$
e\left(G_{y}^{\prime}\right) \int_{G_{y}^{\prime}(F) \backslash G^{\prime}(F)} f^{\prime}\left(h^{-1} y h\right) d h / d u
$$

is equal to

$$
e\left(G_{x}\right) \int_{G_{x}(F) \backslash G(F)} f\left(g^{-1} x g\right) d g / d t
$$

if $y$ comes from $x \in G(F)$ and is equal to 0 if $y$ does not come from any element of $G(F)$. In the formula above the measure $d g$ (resp. $d t$ ) is obtained from the measure $d h$ (resp. $d u$ ) by transporting invariant differentials of top degree from $G^{\prime}$ to $G$ (resp. from $G_{y}^{\prime}$ to $G_{x}$ ); note that we can use the inner twisting $\varphi: G \rightarrow G^{\prime}$ to get an inner twisting $G_{x} \rightarrow G_{y}^{\prime}$, canonical up to inner automorphisms of $G_{y}^{\prime}$. This matching result for functions $f, f^{\prime}$ is stated in slightly different terms on p. 246 of [2]. However the -1 that occurs for central elements is omitted there (formula (8.8) should be replaced by $f(e)=-\varphi(e))$. The same matching result holds when $D$ is replaced by a division algebra of dimension 9 and $G L_{2}$ is replaced by $G L_{3}$ (see Theorem 2.12 of [1] and observe that $e\left(G_{x}\right)$ and $e\left(G_{y}^{\prime}\right)$ are always 1 in this case). 
The generalizations of these two examples (base change, inner twistings) to arbitrary connected reductive groups will have to take into account the difference between conjugacy and stable conjugacy [6], and thus will be more complicated. Nevertheless, it seems likely that the signs that occur will be of the form $e(H)$ where $H$ is some centralizer or twisted centralizer. Moreover it may be the case that the signs $e\left(G_{x}\right)$ will be needed in order to define stable orbital integrals associated to singular semisimple elements.

The remainder of this paper will be devoted to constructing $e(G)$ cohomologically and to proving the functorial properties satisfied by $e(G)$. In order to make the proofs more transparent, we will make our cohomological construction in greater generality. For any field $F$ denote by $B_{2}(F)$ the subgroup of the Brauer group of $F$ consisting of elements $x$ such that $2 x=0$. For any connected reductive group $G$ over $F$, we will define an element $e(G)$ of $B_{2}(F)$. For a nonarchimedean local field, the Brauer group is isomorphic to $\mathbf{Q} / \mathbf{Z}$, hence $B_{2}(F) \simeq\{ \pm 1\}$. The Brauer group of $\mathbf{R}$ is $\{ \pm 1\}$, hence $B_{2}(\mathbf{R}) \simeq\{ \pm 1\}$. The Brauer group of $\mathbf{C}$ is trivial, hence $B_{2}(\mathbf{C}) \simeq\{1\}$. In this way we see that for groups $G$ over a local field $F, e(G)$ can be regarded as an element of $\{ \pm 1\}$.

Here is the construction of $e(G)$. Choose a quasi-split inner form $G^{\prime}$ of $G$ and an inner twisting $\varphi: G \rightarrow G^{\prime}$, that is, an isomorphism $\varphi: G \rightarrow G^{\prime}$ over a separable closure $F_{s}$ of $F$ such that for every $\sigma \in \operatorname{Gal}\left(F_{s} / F\right)$ the automorphism $a_{\sigma}=\varphi^{\sigma} \circ \varphi^{-1}$ of $G^{\prime}$ is inner. Then $\left(a_{\sigma}\right)$ is a 1-cocycle of $\operatorname{Gal}\left(F_{s} / F\right)$ in the adjoint group $G_{\text {ad }}^{\prime}$ of $G^{\prime}$. This gives us an element of $H^{1}\left(\operatorname{Gal}\left(F_{s} / F\right), G_{\mathrm{ad}}^{\prime}\left(F_{s}\right)\right)$. This pointed set is canonically isomorphic to the (nonabelian) étale cohomology set $H_{e}^{1}\left(\operatorname{Spec}(F), G_{\text {ad }}^{\prime}\right)$. We have a map

$$
H_{e}^{1}\left(\operatorname{Spec}(F), G_{\mathrm{ad}}^{\prime}\right) \rightarrow H_{f}^{1}\left(\operatorname{Spec}(F), G_{\mathrm{ad}}^{\prime}\right)
$$

where $H_{f}^{1}\left(\operatorname{Spec}(F), G_{\text {ad }}^{\prime}\right)$ is the nonabelian cohomology set obtained by using the flat topology considered by Shatz on p. 204 of [7]. Consider the sequence

$$
1 \rightarrow Z \rightarrow H \rightarrow G_{\text {ad }}^{\prime} \rightarrow 1
$$

where $H \rightarrow G_{\mathrm{ad}}^{\prime}$ is the simply connected cover of $G_{\mathrm{ad}}^{\prime}$ and $Z$ is the kernel of $H \rightarrow G_{\mathrm{ad}}^{\prime}$ (in other words, the center of $H$ in the sense of group schemes). In general this sequence is not an exact sequence of sheaves in the étale topology on $\operatorname{Spec}(F)$, although it is when the characteristic of $F$ is 0 , or even when the characteristic of $F$ is a prime $p$ and $p$ does not divide the order of the finite group scheme $Z$. However the sequence is an exact sequence of sheaves in the flat topology on $\operatorname{Spec}(F)$ (that $H \rightarrow G_{\text {ad }}^{\prime}$ is an epimorphism of sheaves of sets follows from the fact that the morphism $H \rightarrow G_{\text {ad }}^{\prime}$ is flat and finite). This explains why we are using the flat topology. If we had limited ourselves to fields of characteristic 0 , the étale topology would have been good enough, and we could have used Galois cohomology sets. For the theory of nonabelian cohomology we refer the reader to [3]. Since $Z$ is central in $H$, we have a coboundary map

$$
H_{f}^{\prime}\left(F, G_{\mathrm{ad}}^{\prime}\right) \rightarrow H_{f}^{2}(F, Z)
$$


(we will simplify our notation by writing $F$ instead of $\operatorname{Spec}(F)$ in the cohomology sets). Choose a maximal torus $T$ of $H$ and choose a Borel subgroup $B$ of $H$ containing $T$. Let $\rho$ be half of the sum of the positive roots of $T$ (positive with respect to $B$ ). Since $H$ is simply connected, $\rho$ is a character of $T$. The restriction $\lambda$ of $\rho$ to $Z$ is independent of $T$ and $B$. From this it follows that $\lambda$ is defined over $F$ and that $\lambda$ is preserved by any automorphism of $H$. The character $\lambda: Z \rightarrow \mathbf{G}_{m}$ induces a homomorphism

$$
H_{f}^{2}(F, Z) \rightarrow H_{f}^{2}\left(F, \mathbf{G}_{m}\right) .
$$

But by Theorem 43 on p. 215 of [7], we have $H_{e}^{2}\left(F, \mathbf{G}_{m}\right) \tilde{\rightarrow} H_{f}^{2}\left(F, \mathbf{G}_{m}\right)$, and it is well known that $H_{e}^{2}\left(F, \mathbf{G}_{m}\right)$ is canonically isomorphic to the Brauer group of $F$. Since $2 \lambda$ is trivial on $Z$, the image of $H_{f}^{2}(F, Z)$ in the Brauer group is contained in $B_{2}(F)$. Applying the maps (1), (2), (3) to the element of $H_{e}^{1}\left(F, G_{\mathrm{ad}}^{\prime}\right)$ obtained from the inner twisting $\varphi$, we get an element $e(G)$ of $B_{2}(F)$.

If we make a different choice of $\varphi$, the element of $H_{e}^{1}\left(F, G_{\mathrm{ad}}^{\prime}\right)$ we obtain may be different, but we will now show that the image in $B_{2}(F)$ does not change. Suppose that $\varphi$ is replaced by $\psi: G \rightarrow G^{\prime}$. Write $\psi=\alpha \circ \varphi$ with $\alpha \in$ Aut $_{F_{s}}\left(G^{\prime}\right)$. The condition that $\psi^{\sigma} \circ \psi^{-1}$ be inner for all $\sigma \in \operatorname{Gal}\left(F_{s} / F\right)$ is equivalent to the condition that $\alpha^{\sigma} \circ \alpha^{-1}$ be inner for all $\sigma \in \operatorname{Gal}\left(F_{s} / F\right)$. Since $G^{\prime}$ is quasi-split over $F$ we can find a splitting of the exact sequence

$$
1 \rightarrow G_{\mathrm{ad}}^{\prime}\left(F_{s}\right) \rightarrow \operatorname{Aut}_{F_{s}}\left(G^{\prime}\right) \rightarrow \operatorname{Out}\left(G^{\prime}\right) \rightarrow 1
$$

that is compatible with the action of $\operatorname{Gal}\left(F_{s} / F\right)$. Hence there exists $\beta \in \operatorname{Aut}_{F}\left(G^{\prime}\right)$ such that $\alpha=\beta \circ \gamma$ with $\gamma$ inner. Then

$$
\psi^{\sigma} \circ \psi^{-1}=\beta \circ\left(\gamma^{\sigma} \circ a_{\sigma} \circ \gamma^{-1}\right) \circ \beta^{-1} .
$$

But $\left(\gamma^{\sigma} \circ a_{\sigma} \circ \gamma^{-1}\right)$ is cohomologous to $\left(a_{\sigma}\right)$, and thus the elements of $H_{e}^{1}\left(F, G_{\mathrm{ad}}^{\prime}\right)$ obtained from $\psi$ and $\varphi$ are in the same orbit under the action of $\operatorname{Aut}_{F}\left(G_{\mathrm{ad}}^{\prime}\right)$. But Aut $_{F}\left(G_{\text {ad }}^{\prime}\right)$, which is the same as $\operatorname{Aut}_{F}(H)$, acts on the whole sequence

$$
1 \rightarrow Z \rightarrow H \rightarrow G_{\text {ad }}^{\prime} \rightarrow 1,
$$

hence the elements of $H_{f}^{2}(F, Z)$ obtained from $\psi$ and $\varphi$ are in the same orbit under the action of $\operatorname{Aut}_{F}(H)$. But we have already remarked that the character $\lambda: Z \rightarrow \mathbf{G}_{m}$ is preserved by $\operatorname{Aut}_{F}(H)$. This implies that the elements of $H_{f}^{2}\left(F, \mathbf{G}_{m}\right)$ obtained from $\psi$ and $\varphi$ are the same. This completes the definition of the element $e(G)$ of $B_{2}(F)$. The next step is to establish the functorial properties of $e(G)$.

Proposition. The following properties are satisfied by e $(G)$.

(1) If $G$ is quasi-split, then $e(G)=1$.

(2) For any central subgroup $Z$ of $G$ we have $e(G)=e(G / Z)$.

(3) $e\left(G_{1} \times G_{2}\right)=e\left(G_{1}\right) e\left(G_{2}\right)$ (using multiplicative notation in the Brauer group).

(4) Let $E$ be an extension field of $F$. Then for any $G$ over $F$ we have $e\left(G_{E}\right)=$ $\operatorname{Res}_{E / F}(e(G))$ where $\operatorname{Res}_{E / F}$ denotes the restriction homomorphism from the Brauer group of $F$ to the Brauer group of $E$.

(5) Let $E$ be a finite separable extension field of $F$. Then for any $G$ over $E$ we have $e(R(G))=\operatorname{Cor}_{E / F}(e(G))$ where $R(G)$ denotes the F-group obtained from $G$ by 
restriction of scalars from $E$ to $F$ and $\operatorname{Cor}_{E / F}$ denotes the corestriction homomorphism from the Brauer group of $E$ to the Brauer group of $F$.

(6) Let $S$ be an F-split torus of $G$ and let $M$ be the centralizer of $S$ in $G$. Then $e(G)=e(M)$.

(7) Let $D$ be a central simple algebra over $F$ of dimension $d^{2}$ and let $G$ be the F-group $D^{\times}$. Let $[D]$ denote the class of $D$ in the Brauer group of $F$. If $d$ is odd, then $e(G)$ is trivial. If $d$ is even, then $e(G)=[D]^{d / 2}$ (using multiplicative notation in the Brauer group of $F$ ).

(1), (2), (3), (4) are all obvious. To establish (5) we consider the following commutative diagram

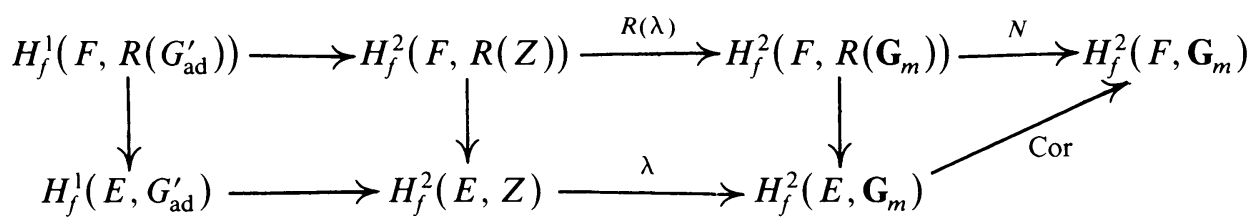

where the vertical arrows are Shapiro maps, obtained as compositions

$$
H_{f}^{1}(F, R(*)) \rightarrow H_{f}^{1}(E, R(*)) \rightarrow H_{f}^{1}(E, *)
$$

(note that over $E$ there is a canonical homomorphism $R(*) \rightarrow *$ ). It is easy to see that $R(Z)$ is the center of $R(H)$, that $R(H)$ is the simply connected cover of $R(G)_{\mathrm{ad}}^{\prime}=$ $R\left(G_{\mathrm{ad}}^{\prime}\right)$, and that the character of $R(Z)$ used to define $e(R(G))$ is the composition

$$
R(Z) \stackrel{R(\lambda)}{\rightarrow} R\left(\mathbf{G}_{m}\right) \stackrel{N}{\rightarrow} \mathbf{G}_{m}
$$

where $N$ is the norm homomorphism. Choose an inner twisting $\varphi: R(G) \rightarrow R\left(G^{\prime}\right)$. Since $G$ is a direct factor (in a canonical way) of $R(G)$ over $E, \varphi$ induces an inner twisting $\psi: G \rightarrow G^{\prime}$. The element of $H_{f}^{1}\left(F, R\left(G_{\mathrm{ad}}^{\prime}\right)\right)$ corresponding to $\varphi$ maps to the element of $H_{f}^{\mathrm{l}}\left(E, G_{\mathrm{ad}}^{\prime}\right)$ corresponding to $\psi$. Hence there is an element of $H_{f}^{2}\left(F, R\left(\mathbf{G}_{m}\right)\right)$ that maps under $N$ to $e(R(G))$ and under the Shapiro map to $e(G)$. The same statement is true with flat cohomology replaced by étale cohomology, or in other words, by Galois cohomology. But by Proposition 8 on p. 33 of [7], the corestriction homomorphism from $H^{2}\left(E, \mathbf{G}_{m}\right)$ to $H^{2}\left(F, \mathbf{G}_{m}\right)$ is the composition of $N$ with the inverse of the Shapiro isomorphism. This proves (5).

Next we prove (6). Let $S_{\text {ad }}$ denote the image of $S$ in $G_{\text {ad }}$ and let $C$ denote the center of $G$. Then the centralizer of $S_{\text {ad }}$ in $G_{\text {ad }}$ is equal to the image of $M$ in $G_{\text {ad }}$ and is therefore isomorphic to $M / C$. By (2) we have $e(M)=e(M / C)$ and $e(G)=$ $e(G / C)$. This reduces us to the case in which $G$ is an adjoint group.

Our next step is to show that the inner twisting $\varphi: G \rightarrow G^{\prime}$ can be chosen so that the restriction of $\varphi$ to $S$ is defined over $F$. For this we may as well assume (temporarily) that $S$ is a maximal $F$-split torus of $G$. Choose a minimal parabclic $F$-subgroup $P$ of $G$ of which $M=\operatorname{Cent}_{G}(S)$ is a Levi subgroup. Choose a maximal $F$-split torus $S^{\prime}$ of $G^{\prime}$ and a Borel subgroup $B^{\prime}$ of $G^{\prime}$ defined over $F$ of which $T^{\prime}=$ Cent $_{G^{\prime}}\left(S^{\prime}\right)$ is a Levi subgroup $\left(T^{\prime}\right.$ is a maximal torus of $\left.G^{\prime}\right)$. Choose also a maximal $F$-torus $T$ of $M$; then $T$ is a maximal $F$-torus of $G$ that contains $S$. We may 
choose the inner twisting $\varphi: G \rightarrow G^{\prime}$ so that $\varphi(T)=T^{\prime}$ and $\varphi(P) \supset B^{\prime}$. With these assumptions $\left.\varphi\right|_{S}$ is defined over $F$, as we will now show. Let $\sigma \in \operatorname{Gal}\left(F_{s} / F\right)$. Let $g_{\sigma}$ be an element of $G^{\prime}$ such that $\varphi \circ \varphi^{-\sigma}=\operatorname{Int}\left(g_{\sigma}\right)$. It follows from $\varphi(P) \supset B^{\prime}$ that (Int $\left.g_{\sigma}\right)\left(B^{\prime}\right) \subset \varphi(P)$. This means that (Int $\left.g_{\sigma}\right)\left(B^{\prime}\right)$ and $B^{\prime}$ are two Borel subgroups contained in $\varphi(P)$, and hence that $g_{\sigma} \in \varphi(P)$. It follows from $\varphi(T)=T^{\prime}$ that $g_{\sigma}$ normalizes $T^{\prime}$. These two conditions on $g_{\sigma}$ imply that $g_{\sigma}$ belongs to the unique Levi component of $\varphi(P)$ containing $T^{\prime}$, namely $\varphi(M)$. In particular $g_{\sigma}$ centralizes $\varphi(S)$ and therefore the restriction of $\varphi$ to $S$ is defined over $F$.

From now on we assume that $\left.\varphi\right|_{S}$ is defined over $F$. Let $M^{\prime}$ denote the centralizer of $\varphi(S)$. This usage of notation is consistent with our previous usage since $M^{\prime}$ is in fact a quasi-split inner form of $M$. Let $p: H \rightarrow G^{\prime}$ be a simply connected covering of $G^{\prime}$, and let $Z$ be the kernel of $p$. The sequence $1 \rightarrow Z \rightarrow H \rightarrow G^{\prime} \rightarrow 1$ and the character $\lambda: Z \rightarrow \mathbf{G}_{m}$ defined previously are what we need to calculate $e(G)$. Let $I$ denote the inverse image of $M^{\prime}$ under $p$. It is not hard to show that $I$ is the centralizer in $H$ of the $F$-split torus $p^{-1}(\varphi(S))$. Hence $I$ is a Levi subgroup of a parabolic $F$-subgroup of $H$, and in particular the derived group $I_{\text {der }}$ of $I$ is simply connected (because $H$ is simply connected). Let $Z_{M}$ denote the center of $I_{\mathrm{der}}$. The sequence

$$
1 \rightarrow Z_{M} \rightarrow I_{\mathrm{der}} \rightarrow M_{\mathrm{ad}}^{\prime} \rightarrow 1
$$

and character $\lambda_{M}: Z_{M} \rightarrow \mathbf{G}_{m}$ are what we need to calculate $e(M)$. The reason for assuming that the restriction of $\varphi$ to $S$ is defined over $F$ is that this condition implies that the element of $H_{e}^{1}\left(F, G^{\prime}\right)$ corresponding to $\varphi$ lies in the image of $H_{e}^{1}\left(F, M^{\prime}\right)$. The same is then true for the flat cohomology sets. A consequence of this is that the element of $H_{f}^{2}(F, Z)$ used to get $e(G)$ can be calculated using the coboundary map associated to the sequence

$$
1 \rightarrow Z \rightarrow I \rightarrow M^{\prime} \rightarrow 1 .
$$

Both $Z$ and $Z_{M}$ are subgroups of Center( $\left.I\right)$. By considering the sequences (A) and (B) above together with the sequence

$$
1 \rightarrow \operatorname{Center}(I) \rightarrow I \rightarrow M_{\mathrm{ad}}^{\prime} \rightarrow 1
$$

we see that the elements of $H_{f}^{2}(F, Z)$ and $H_{f}^{2}\left(F, Z_{M}\right)$ used to obtain $e(G)$ and $e(M)$ respectively have the same image in $H_{f}^{2}(F, \operatorname{Center}(I))$. To complete the proof it is enough to show that there is a character defined over $F$ on $\operatorname{Center}(I)$ that restricts to $\lambda$ on $Z$ and to $\lambda_{M}$ on $Z_{M}$. Choose a maximal $F$-split torus $S_{0}$ of $H$ containing $p^{-1}(\varphi(S))$ and let $T_{0}$ be the centralizer of $S_{0}$ in $H$. Since $H$ is quasi-split, $T_{0}$ is a maximal $F$-torus of $H$ and there exists a Borel subgroup $B_{0}$ of $H$ defined over $F$ containing $T_{0}$. Let $\rho$ be half the sum of the positive roots on $T_{0}$. Then $\rho$ is defined over $F$, and the restriction of $\rho$ to $Z$ is by definition $\lambda$. Because we chose $S_{0}$ so as to have it contain $p^{-1}(\varphi(S))$, we have that $T_{0}$ is a maximal torus of $I$. Hence Center $(I)$ is contained in $T_{0}$ and we may restrict $\rho$ to $\operatorname{Center}(I)$ to get a character of that group defined over $F$ whose restriction to $Z$ is $\lambda$. An easy calculation using the expression for $\rho$ as the sum of the fundamental weights of $T_{0}$ shows that the restriction of $\rho$ to $Z_{M}$ is $\lambda_{M}$ (the fundamental weights of $H$ that are not fundamental weights of $I$ are trivial on $Z_{M}$ ). This completes the proof of (6). 
The last statement to prove is (7). The quasi-split inner form of $G$ is $G^{\prime}=G L_{d}$. The sequence used to compute $e(G)$ is

$$
1 \rightarrow \mu_{d} \rightarrow S L_{d} \rightarrow P G L_{d} \rightarrow 1 .
$$

The sequence

$$
1 \rightarrow \mu_{d} \rightarrow \mathbf{G}_{m} \stackrel{d}{\rightarrow} \mathbf{G}_{m} \rightarrow 1
$$

is an exact sequence of sheaves in the flat topology. Since $H_{f}^{1}\left(F, \mathbf{G}_{m}\right)=H_{e}^{1}\left(F, \mathbf{G}_{m}\right)$ $=\{0\}$, the long exact cohomology sequence of $(*)$ shows that $H_{f}^{2}\left(F, \mu_{d}\right)$ may be identified with the elements of the Brauer group killed by $d$. If we use an inner twisting $\varphi: G \rightarrow G^{\prime}$ obtained from an isomorphism $D \otimes_{F} F_{s} \tilde{\rightarrow} M_{d}\left(F_{s}\right)$, we find that the element of $H_{f}^{2}(F, Z)$ associated to $\varphi$ is [ $D$ ], the class of $D$ in the Brauer group of $F$. The character $\lambda: \mu_{d} \rightarrow \mathbf{G}_{m}$ is easy to calculate. It is trivial when $d$ is odd and is given by $z \mapsto z^{d / 2}$ when $d$ is even. This proves statement (7).

Using well-known facts about the Brauer groups of local fields we obtain the following result.

COROLlaRY. Over a local field $F$ the following properties are satisfied by $e(G)$, with $e(G)$ now viewed as an element of $\{ \pm 1\}$.

(1) If $G$ is quasi-split, then $e(G)=1$.

(2) For any central subgroup $Z$ of $G$ we have $e(G)=e(G / Z)$.

(3) $e\left(G_{1} \times G_{2}\right)=e\left(G_{1}\right) e\left(G_{2}\right)$.

(4) Let $E$ be a finite extension field of $F$. Then for any $G$ over $F$ we have $e\left(G_{E}\right)=e(G)^{[E: F]}$.

(5) Let $E$ be a finite separable extension field of $F$. Then for any $G$ over $E$ we have $e\left(\operatorname{Res}_{E / F} G\right)=e(G)$.

(6) Let $S$ be an F-split torus of $G$ and let $M=\operatorname{Cent}_{G}(S)$. Then $e(G)=e(M)$.

(7) Let $D$ be a central simple algebra over $F$ of dimension $d^{2}$ with invariant $r / d \in \mathbf{Q} / \mathbf{Z}$ and let $G$ be the $F$-group $D^{\times}$. Then $e(G)$ is trivial unless $d$ is even and $r$ is odd, in which case $e(G)=-1$.

We are now in a position to show that $e(G)$ has the values given previously. For $F=\mathbf{C}$ the Brauer group is trivial and hence $e(G)=1$ for all $G$. For $F=\mathbf{R}$ we define a quantity $e^{\prime}(G)$ by the formula

$$
e^{\prime}(G)=(-1)^{q\left(G^{\prime}\right)-q(G)} .
$$

We must prove that $e(G)=e^{\prime}(G)$ for all $G$. Let $S$ be a maximal R-split torus of $G$ and denote by $M$ the centralizer of $S$ in $G$. Then $e(G)=e(M)$ by (6) of the corollary above. By Proposition 6.6 of $[8]$ we have

$$
q\left(G^{\prime}\right)-q(G)=q\left(M^{\prime}\right)-q(M)
$$

(this proposition is proved by showing that $q(G)-q(M)=\frac{1}{2}\left[\operatorname{dim}_{\mathbf{C}} N+\operatorname{dim}_{\mathrm{C}} S\right.$ ] where $N$ is the unipotent radical of a parabolic subgroup of $G$ with Levi component $M$, and noting that $\operatorname{dim}_{\mathrm{C}} N$ and $\operatorname{dim}_{\mathrm{C}} S$ do not change when $G, M$ are replaced by $\left.G^{\prime}, M^{\prime}\right)$. Thus we have $e^{\prime}(G)=e^{\prime}(M)$, and it suffices to show that $e(M)=e^{\prime}(M)$. 
We further have that $e(M)=e\left(M_{\mathrm{ad}}\right)$ and $e^{\prime}(M)=e^{\prime}\left(M_{\mathrm{ad}}\right)$. Since $M_{\mathrm{ad}}$ is anisotropic, we have reduced our problem to the case in which $G$ is an anisotropic adjoint group over $\mathbf{R}$.

Since $G^{\prime}$ is an inner twist of an anisotropic group, $G^{\prime}$ has a maximal $\mathbf{R}$-torus $T^{\prime}$ that is anisotropic over $\mathbf{R}$ and there exists an element $s \in T^{\prime}(\mathbf{R})$ with $s^{2}=1$ such that $\operatorname{Int}(s)$ is a Cartan involution of $G^{\prime}$. Let $G_{s}^{\prime}$ denote the centralizer of $s$ in $G^{\prime}$. Then $G_{s}^{\prime}(\mathbf{R})$ is a maximal compact subgroup of $G^{\prime}(\mathbf{R})$. We have $q(G)=0$ since $G$ is anisotropic, and we have

$$
q\left(G^{\prime}\right)=\frac{1}{2}\left(\operatorname{dim}_{\mathbf{C}} G^{\prime}-\operatorname{dim}_{\mathbf{C}} G_{s}^{\prime}\right)=\frac{1}{2}\left(\operatorname{dim} \operatorname{Lie}\left(G^{\prime}\right)-\operatorname{dim} \operatorname{Lie}\left(G_{s}^{\prime}\right)\right) .
$$

Let $R$ denote the set of roots of $T^{\prime}$ in $G^{\prime}$. Then

$$
\operatorname{dim} \operatorname{Lie}\left(G^{\prime}\right)-\operatorname{dim} \operatorname{Lie}\left(G_{s}^{\prime}\right)=\operatorname{Card}\{\alpha \in R: \alpha(s) \neq 1\} .
$$

Choose an ordering on $R$ and let $R_{+}$denote the set of positive roots with respect to this ordering. Let

$$
q=\operatorname{Card}\left\{\alpha \in R_{+}: \alpha(s) \neq 1\right\}
$$

We need to show that $e(G)=(-1)^{q}$.

The inner form $G$ of $G^{\prime}$ can be obtained from the 1-cocycle $\left(c_{\sigma}\right)$ where $c_{\sigma}=1$ if $\sigma$ is trivial and $c_{\sigma}=s$ if $\sigma$ is complex conjugation. Since the characteristic of $\mathbf{R}$ is 0 , we may calculate $e(G)$ using Galois cohomology instead of flat cohomology. As usual denote by $Z$ the center of the simply connected cover $H$ of $G^{\prime}$. Choose an element $t \in H(\mathbf{R})$ that maps to $s$ under $H \rightarrow G^{\prime}$ (the existence of $t$ follows from the connectedness of $\left.T^{\prime}(\mathbf{R})\right)$. The image of $\left(c_{\sigma}\right)$ under $H^{1}\left(\mathbf{R}, G^{\prime}\right) \rightarrow H^{2}(\mathbf{R}, Z)$ can be represented by $\left(d_{\sigma \tau}\right)$ where $d_{\sigma \tau}=1$ unless $\sigma, \tau$ are both complex conjugation in which case $d_{\sigma \tau}=t^{2}$. The image of $\left(d_{\sigma \tau}\right)$ under the homomorphism

$$
H^{2}(\mathbf{R}, Z) \rightarrow H^{2}\left(\mathbf{R}, \mathbf{G}_{m}\right)
$$

induced by $\lambda$ can be represented by $\left(e_{\sigma \tau}\right)$ where $e_{\sigma \tau}=\lambda\left(d_{\sigma \tau}\right)$. We have

$$
\lambda\left(t^{2}\right)=\lambda^{2}(t)=\prod_{\alpha \in R_{+}} \alpha(t)=\prod_{\alpha \in R_{+}} \alpha(s) .
$$

But since $s^{2}=1$ the only possible values for $\alpha(s)$ are 1 and -1 , and thus it follows that $\lambda\left(t^{2}\right)=(-1)^{q}$. This shows that $e_{\sigma \tau}=1$ unless $\sigma, \tau$ are both complex conjugation, in which case $e_{\sigma \tau}=(-1)^{q}$. For $q=1$ this 2-cocycle represents the nontrivial element of $H^{2}\left(\mathbf{R}, \mathbf{G}_{m}\right)=\{+1\}$ (it must be nontrivial since there are groups for which $e(G)=-1)$. This shows that $e(G)=(-1)^{q}$.

We will use a similar method for nonarchimedean local fields $F$. In this case we define a quantity $e^{\prime}(G)$ by the formula

$$
e^{\prime}(G)=(-1)^{r\left(G^{\prime}\right)-r(G)} \text {. }
$$

We must show that $e(G)=e^{\prime}(G)$ for all $G$. Let $S$ be a maximal $F$-split torus of $G$ and denote by $M$ the centralizer of $S$ in $G$. We know that $e(G)=e(M)$. But $r(G)-r(M)$ is equal to the difference of the $F$-ranks of $\operatorname{Center}(M)$ and $\operatorname{Center}(G)$, hence does not change when $G, M$ are replaced by $G^{\prime}, M^{\prime}$; therefore we also have $e^{\prime}(G)=e^{\prime}(M)$. As for real groups we are reduced to the case in which the derived 
group of $G$ is anisotropic. We may assume that $G$ is adjoint as well (since $e(G)$ and $e^{\prime}(G)$ both depend only on $\left.G_{\mathrm{ad}}\right)$. Since $e$ and $e^{\prime}$ are multiplicative for direct products of groups, we may assume that $G$ is $F$-simple. Then $G$ is isomorphic to a group of the form $\operatorname{Res}_{E / F} H$ for some finite separable extension $E$ of $F$ and some absolutely simple $E$-group $H$. We have $e(G)=e(H)$. Since $r(G)=r(H)$ and $r\left(G^{\prime}\right)=r\left(H^{\prime}\right)$ we also have $e^{\prime}(G)=e^{\prime}(H)$. This reduces us to the case in which $G$ is absolutely simple as well as anisotropic and adjoint. With these conditions $G$ must be isomorphic to $D^{\times} / F^{\times}$for some central division algebra $D$ over $F$. Write $\operatorname{inv}(D)=r / d$ for relatively prime positive integers $r, d$. Then $r(G)=0$ and $r\left(G^{\prime}\right)=r\left(P G L_{d}\right)=d-1$. This shows that $e^{\prime}(G)=(-1)^{d-1}$. By statement $(7)$ of the corollary above we also have $e(G)=(-1)^{d-1}$.

Our last result is a product formula.

Proposition. Let $F$ be a global field and let $S$ be the set of places of $F$. For $v \in S$ let $G_{v}$ denote the $F_{v}$-group obtained from $G$ by extension of scalars from $F$ to $F_{v}$. Then $e\left(G_{v}\right)=1$ for all but a finite number of $v \in S$, and

$$
\prod_{v \in S} e\left(G_{v}\right)=1
$$

We first note that $G_{v}$ is quasi-split for all but a finite number of $v \in S$, and hence that $e\left(G_{v}\right)=1$ for all but a finite number of $v \in S$. Let $D$ be a central division algebra over $F$ whose class in the Brauer group of $F$ is equal to $e(G)$. It follows from (4) of the proposition proved earlier that

$$
e\left(G_{v}\right)=\exp \left(2 \pi i \cdot \operatorname{inv}\left(D_{v}\right)\right) .
$$

The product formula then follows from the well-known formula

$$
\sum_{v \in S} \operatorname{inv}\left(D_{v}\right)=0
$$

\section{REFERENCES}

1. D. Flath, A comparison of the automorphic representations of GL(3) and its twisted forms, Thesis, Harvard University, 1977; Pacific J. Math. (to appear).

2. S. Gelbart and H. Jacquet, Forms of GL(2) from the analytic point of view, Proc. Sympos. Pure Math., vol. 33, part 1, Amer. Math. Soc., Providence, R.I., 1979, pp. 213-251.

3. J. Giraud, Cohomologie non abélienne, Springer-Verlag, Berlin, Heidelberg and New York, 1971.

4. H. Jacquet and R. P. Langlands, Automorphic forms on GL(2), Lecture Notes in Math., vol. 114, Springer-Verlag, Berlin, Heidelberg and New York, 1970.

5. R. Kottwitz, Orbital integrals and base change, Proc. Sympos. Pure Math., vol. 33, part 2, Amer. Math. Soc., Providence, R.I., 1979, pp. 111-113.

6. R. P. Langlands, Stable conjugacy: definitions and lemmas, Canad. J. Math. 31 (1979), 700-725.

7. S. Shatz, Profinite groups, arithmetic, and geometry, Ann. of Math. Studies, no. 67, Princeton Univ. Press and Univ. of Tokyo Press, 1972.

8. D. Shelstad, Characters and inner forms of a quasi-split group over $\mathbf{R}$, Comp. Math. 39 (1979), 11-45.

Department of Mathematics, University of Washington, Seattle, Washington 98195 